\title{
Software Libre para el Análisis Espectral de Señales
}

\section{Free Software for Spectral Analysis of Signals}

DOI: $10.46932 / \mathrm{sfjdv2n2-070}$

Received in: March 1st, 2021

Accepted in: May 30th, 2021

\author{
Carlos Alberto Vanegas \\ Maestría en Ingeniería \\ Profesor Titular \\ Universidad Distrital Francisco \\ José de Caldas \\ Bogotá-Colombia \\ Facultad Tecnológica \\ cavanegas@udistrital.edu.co \\ Rocío Rodríguez Guerrero \\ Maestría en Ciencias \\ Profesor Asociado \\ Universidad Distrital \\ Francisco José de Caldas \\ Bogotá-Colombia \\ Facultad Tecnológica \\ rrodriguezg@udistrital.edu.co
Gerardo Castang Montiel
Maestría en Teleinformática
Profesor Asociado
Universidad Distrital

\section{RESUMEN}

En el presente artículo se realiza el análisis espectral de señales en particular de algunos de los códigos de línea más utilizados o comunes en la representación eléctrica de una secuencia binaria de datos, con el propósito de validar su comportamiento espectral, así como su dependencia de los parámetros tasa de bit (R) que corresponde a la velocidad a la cual se transmiten los datos, en bits por segundo; frecuencia (f) definida como aquellas bandas o rangos en las que se distribuyen los componentes de potencia, dada en hertz y tiempo de bit ( $\mathrm{Tb}$ ) que corresponde al tiempo que dura la presencia o transmisión de un bit, dada en segundos y su incidencia en las gráficas de la densidad espectral de potencia.

Se utiliza el software libre Python aplicado en casos de análisis de señales y en la enseñanza de temas de ingeniería, particularmente en la telemática y las comunicaciones.

Palabras clave: Códigos de Línea, Análisis Espectral de Señales, Densidad Espectral de Potencia, Representación de Señales. 


\section{ABSTRACT}

In this article the spectral analysis of signals in particular of some of the most used or common line codes in the electrical representation of a binary data sequence is carried out, in order to validate their spectral behavior, as well as their dependence on the parameters bit rate $(\mathrm{R})$ that corresponds to the speed at which the data is transmitted, in bits per second; frequency (f) defined as those bands or ranges in which the power components are distributed, given in hertz and bit time ( $\mathrm{Tb}$ ) that corresponds to the time that the presence or transmission of a bit lasts, given in seconds and its incidence on the power spectral density graphs.

The free software Python applied is used in signal analysis cases and in the teaching of engineering subjects, particularly in telematics and communications.

Keywords: Line Codes, Signal Spectral Analysis, Power Spectral Density, Signal Representation.

\section{INTRODUCCIÓN}

Los códigos de línea permiten realizar la representación de una secuencia de datos a través de pulsos o señales digitales. Para toda señal, es necesario conocer su comportamiento tanto en el dominio del tiempo como en el dominio de la frecuencia. Este análisis permite conocer cuál es el ancho de banda efectivo de la señal, que permita realizar el procesamiento y/o transmisión de la misma.

Realizar la convalidación de la teoría asociada al análisis espectral de señales, posibilita determinar el comportamiento en el dominio de la frecuencia de algunos de los códigos de líneas más utilizados o conocidos como son el unipolar sin retorno a cero (Unipolar NRZ), polar sin retorno a cero (Polar NRZ), unipolar con retorno a cero (Unipolar RZ), bipolar con retorno a cero (Bipolar RZ), codificación bifase (Manchester).

\section{OBJETIVOS}

Se plantea analizar el caso de la densidad espectral de potencia normalizada, cuando el parámetro tasa de bits es igual al parámetro de frecuencia, donde se analizan dos casos: el primero (caso 1-A), para una tasa de bits de un bit por segundo, y el segundo (caso 1-B), para una tasa de bit de mil bits por segundo, pretendiendo observar el efecto de la variación de la tasa de bits de una transmisión de información en las gráficas resultantes de la densidad espectral de potencia de los códigos de línea utilizados.

Así mismo, se define el parámetro de amplitud A en cada ecuación para la obtención de la densidad espectral de potencia que se ajusta para el caso normalizado. En estos casos, el valor de la frecuencia normalizada se multiplica por el valor de $\mathrm{R}$, en el eje $\mathrm{x}$, $\mathrm{y}$ el de la densidad espectral de potencia se multiplica por el valor de $\mathrm{Tb}$, en el eje $\mathrm{y}$.

\section{METODOLOGÍA Y PROCESOS DE DESARROLLO}


Se plantea la obtención y el análisis de las gráficas de la densidad espectral de potencia en el caso normalizado, comparando el efecto de la variación del parámetro tasa de bits (R), analizando cada gráfica según el caso.

Para la obtención de las gráficas de la densidad espectral de potencia normalizada, se utilizan las expresiones correspondientes de la función densidad espectral de potencia definida para los códigos de línea analizados, las cuales se representan en las líneas de código correspondiente en el lenguaje python.

Caso $1-A: R=1 b p s, f=1 H z, T b=1$ segundo; donde $T b=1 / R$, siendo $f=R$ y se especifica el parámetro amplitud A, para el caso normalizado.

Figura 1. Densidad espectral de potencia de una señal con codificación Manchester, $\mathrm{R}=1 \mathrm{bps}$, caso normalizado.

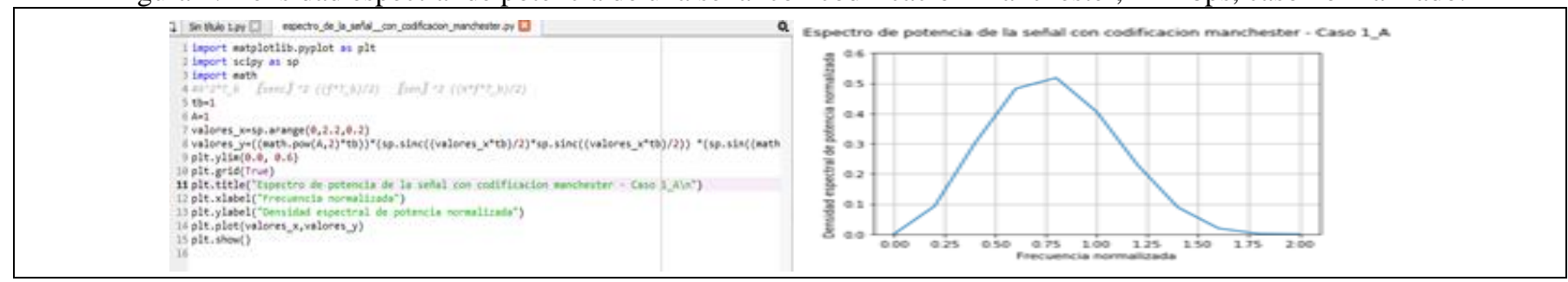

$\mathrm{Al}$ analizar la forma de onda resultante, se evidencia que se encuentra la forma de onda prevista para la señal con codificación Manchester y no existe componente de dc (componente de corriente directa, para $\mathrm{f}=0$ ); en este caso, su señal de densidad espectral de potencia se asocia con una función de distribución de probabilidad gausiana, con su valor máximo alrededor de $\mathrm{f}=0.75$, con un valor de densidad espectral de potencia de 0.5 , para $\mathrm{A}^{\wedge} 2=1$.

Figura 2. Densidad espectral de potencia de una señal con codificación unipolar con retorno a cero.

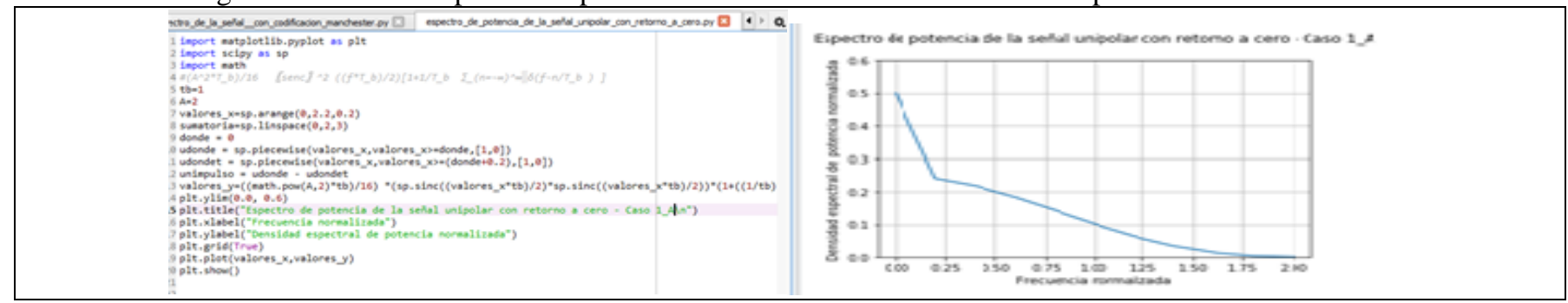

Se cumple con la forma de onda esperada, presenta una función decreciente con su valor máximo en cero hasta dos, y tiene un componente dc en cero, y una función delta de peso o magnitud 0.25 para $\mathrm{f}=0$, con un valor de densidad espectral de 0.5; así mismo, se debe incluir una función delta de peso o magnitud 0.1 para $\mathrm{f}=1$; esto para $\mathrm{A}^{\wedge} 2=4$. 
Figura 3. Densidad espectral de potencia de una señal unipolar sin retorno a cero

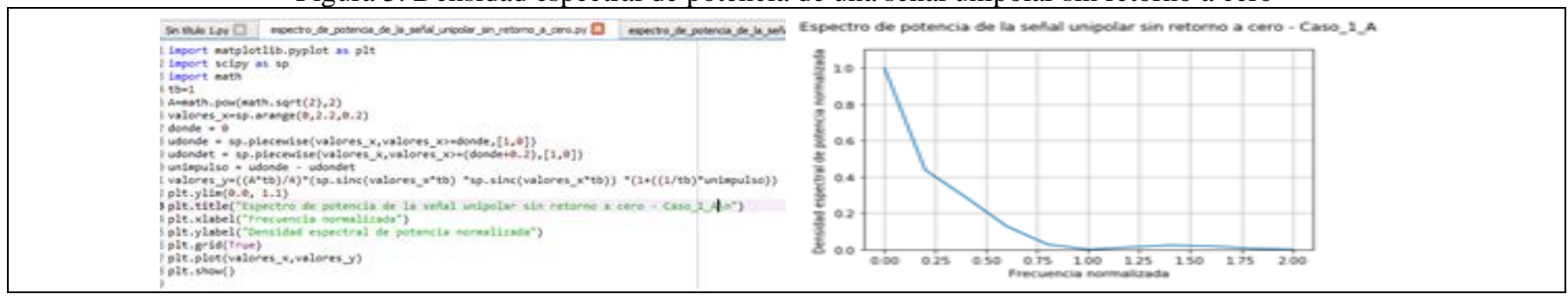

Para este caso, se presenta un componente dc, con un valor máximo de 0.5 en la densidad espectral de potencia y un componente de la función delta para $\mathrm{f}=0$, con peso o magnitud de 0.5 , presentando un cruce por cero alrededor de $\mathrm{f}=1$, para $\mathrm{A}^{\wedge} 2=2$.

Figura 4. Densidad espectral de potencia de la señal bipolar con retorno a cero

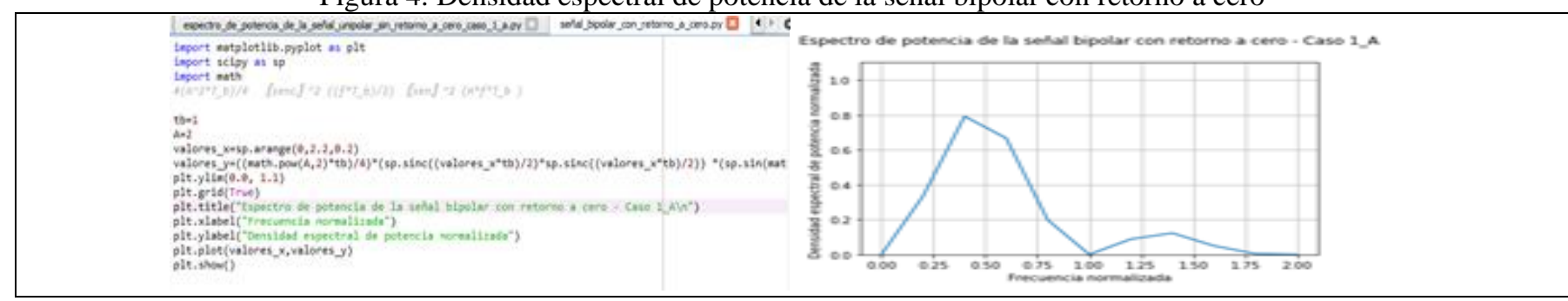

Para este caso, se cumple con la forma de onda esperada de la densidad espectral de potencia, no presenta componente de dc, ni hay componente asociado de la función delta, presentando un cruce por cero para $\mathrm{f}=1$, y su valor máximo alrededor de $\mathrm{f}=0.5$, con un valor cercano a 0.8 , esto para $\mathrm{A}^{\wedge} 2=4$. Además, presenta un incremento o presencia de la densidad espectral de potencia en $\mathrm{f}=1.3$, con una excursión de 0.1 .

Figura 5. Densidad espectral de potencia de la señal polar sin retorno a cero.

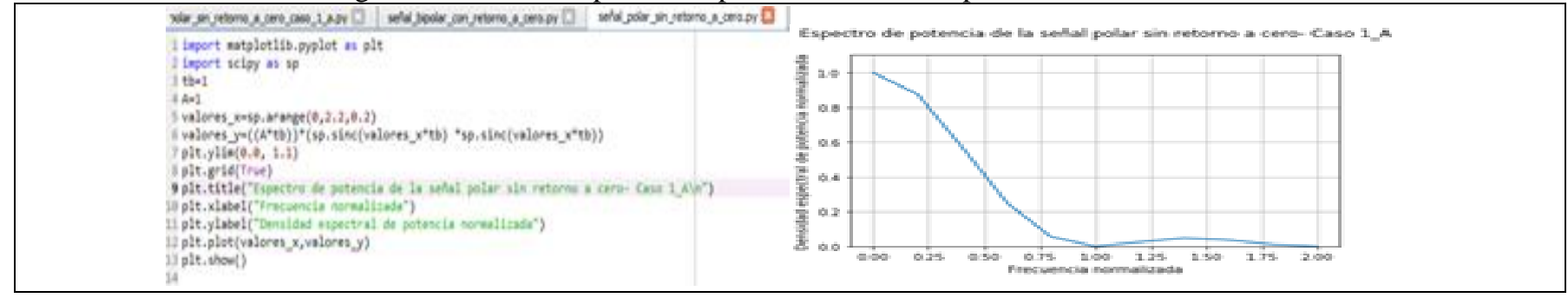

Para este caso, existe componente de $\mathrm{dc}$, para $\mathrm{f}=0$, presentando el valor máximo de salida de la gráfica de la densidad espectral de potencia normalizada; es una función decreciente que presenta un cruce por cero alrededor de $\mathrm{f}=1$, es decir, que los mayores componentes de potencia se encuentran alrededor de $\mathrm{f}=0$ hasta $\mathrm{f}=1$, presentando un leve incremento o presencia de la densidad espectral de potencia entre $\mathrm{f}=1$ y $f=2$. 
Caso 1-B: $R=1000$ bps, $f=1000 \mathrm{~Hz}, T b=1 \mathrm{mseg}=0.001$ segundos. Caso normalizado, donde $f=R$, y se mantiene el valor del parámetro de amplitud A, igual en cada caso.

Figura 6. Densidad espectral de potencia de una señal con codificación manchester, para R=1000 bps, caso normalizado.

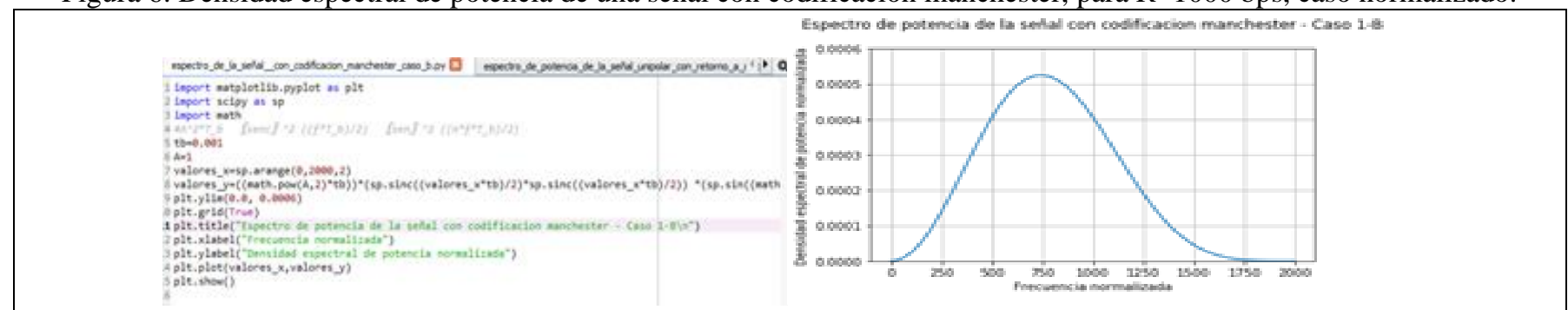

Se identifica una forma de onda más suavizada, o uniforme, con un valor máximo alrededor de $\mathrm{f}=750 \mathrm{~Hz}$, pero el valor máximo de la densidad de potencia normalizada es un poco mayor a 0.0005 , debido a que el valor de $\mathrm{Tb}$ es un milisegundo. El parámetro de amplitud para el caso normalizado se mantiene en $\mathrm{A}^{\wedge} 2=1$.

Figura 7. Densidad espectral de potencia de una señal unipolar con retorno a cero, para R=1000bps, caso normalizado.

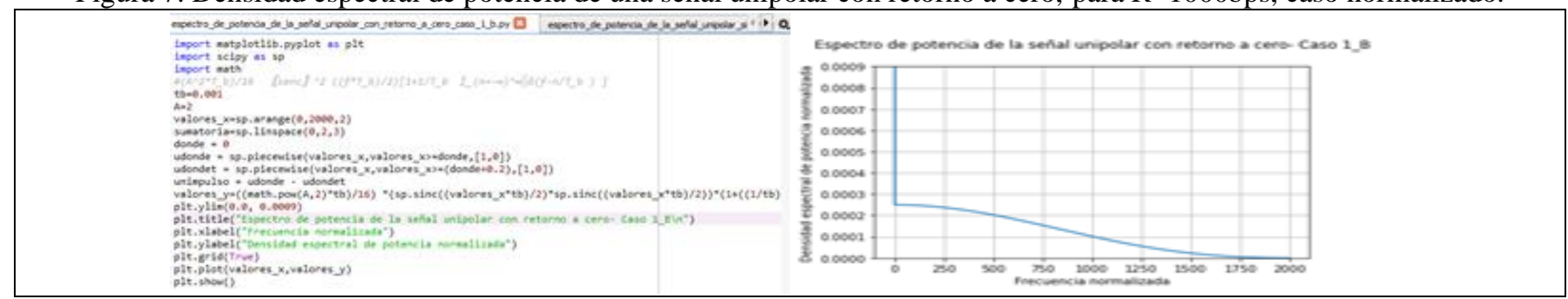

Se evidencia la existencia del componente de dc, así como, la presencia de los componentes de la función delta para $\mathrm{f}=0$, y se debe incluir un componente de la función delta para f=1000; la gráfica de la función de densidad espectral es una señal decreciente, que tiende a cero para f=2R. El valor máximo de la densidad espectral de potencia es cercano a 0.0002. Para este caso, el parámetro de amplitud se mantiene en $\mathrm{A}^{\wedge} 2=4$, para el caso normalizado.

Figura 8. Densidad espectral de potencia de la señal unipolar sin retorno a cero, con $\mathrm{R}=1000$ bps, caso normalizado.

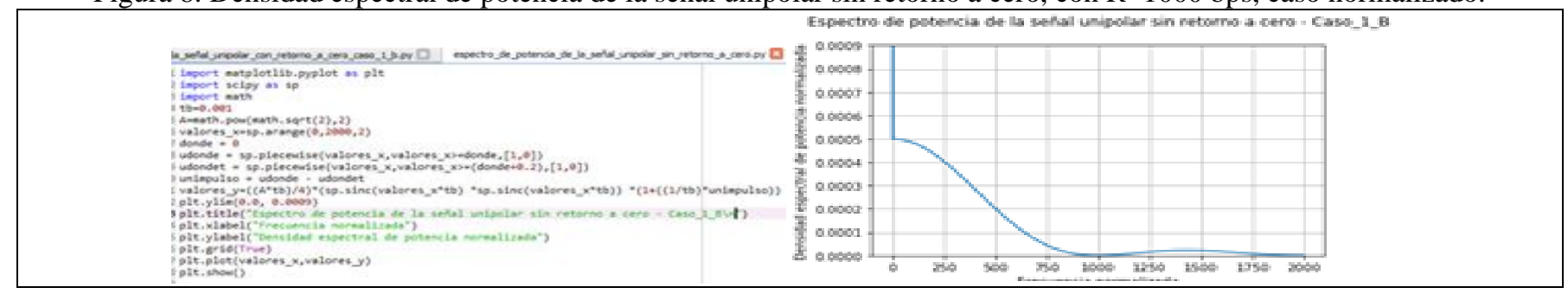


Se presenta una señal suavizada, que presenta un componente de dc, para $\mathrm{f}=0$, de 0.0005 y un componente de la función delta para esta misma frecuencia de magnitud o peso de 0.0005 , presentando un cruce por cero para $\mathrm{f}=1000$, y tendiendo a cero para $\mathrm{f}=2000$, o equivalente a $\mathrm{f}=2 \mathrm{R}$. Es decir, que los componentes significativos de magnitud o de densidad espectral se encuentran en el rango de $f=0$ a $f=1000$ Hz. El parámetro de amplitud $\mathrm{A}^{\wedge} 2=2$.

Figura 9. Densidad espectral de potencia de una señal bipolar con retorno a cero, para $\mathrm{R}=1000 \mathrm{bps}$, caso normalizado.

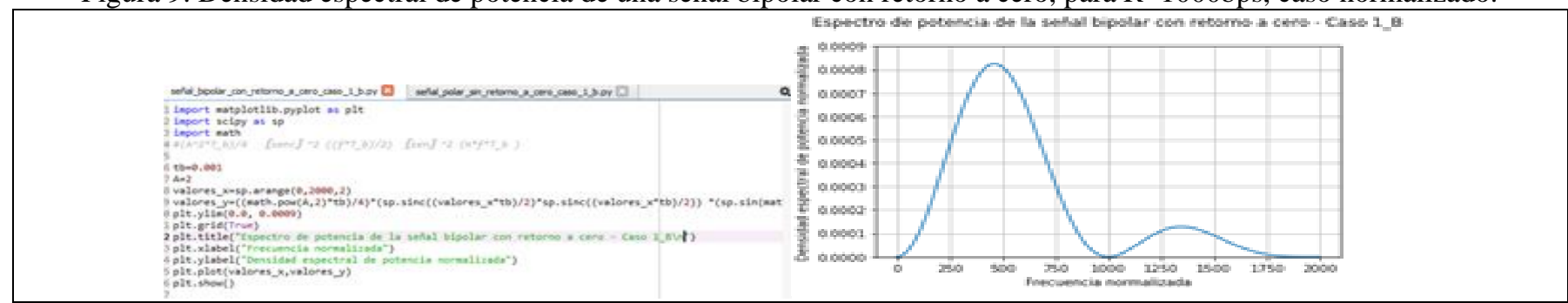

Se encuentra una señal continua, suavizada, con un valor máximo en la densidad espectral de potencia alrededor de 0.0008 , para una frecuencia cercana de $500 \mathrm{~Hz}$, en el rango de 0 a $1000 \mathrm{~Hz}$. Existen otros componentes de potencia de la señal en el rango de $1000 \mathrm{~Hz}$ a $2000 \mathrm{~Hz}$, con una frecuencia cercana a los $1300 \mathrm{~Hz}$, para obtener componente de potencia de señal por encima de 0.0001 . Para este caso $\mathrm{A}^{\wedge} 2=4$.

Esta señal podría filtrarse utilizando un filtro pasabanda entre los $250 \mathrm{~Hz}$ y los $750 \mathrm{~Hz}$, es decir, con un ancho de banda de $500 \mathrm{~Hz}$, para la obtención de los componentes más significativos de la densidad espectral de potencia de la señal. Un filtro pasabanda es aquel filtro que solo deja pasar una banda de frecuencia determinada, particularmente aquella en la que se encuentran los componentes de potencia más relevantes o significativos de la señal.

Figura 10. Densidad espectral de potencia de una señal polar sin retorno a cero, para $\mathrm{R}=1000$ bps, caso normalizado.

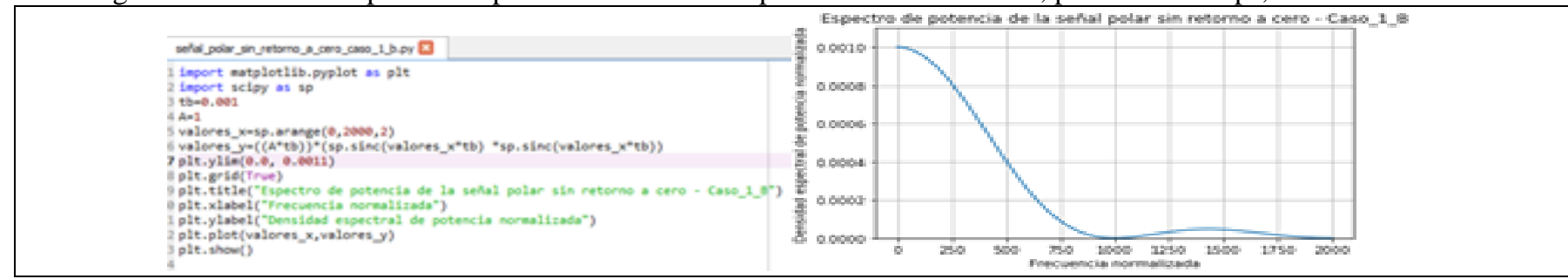

Se muestra una señal continua, con una forma de onda suavizada, que presenta un componente dc para $\mathrm{f}=0$, con un valor máximo de excursión de señal de 0.0001 ; esta señal presenta un cruce por cero para $\mathrm{f}=1000 \mathrm{~Hz}$, y tiende a cero cuando $\mathrm{f}=2000 \mathrm{~Hz}$; los máximos niveles de densidad de potencia de señal se encuentran entre 0 y $500 \mathrm{~Hz}$, siendo la forma de onda un señal decreciente. En este caso $\mathrm{A}^{\wedge} 2=1$. 
Se podría utilizar un filtro pasabajas, entre 0 y $750 \mathrm{~Hz}$, para extraer los componentes significativos de potencia de señal, y no teniendo en cuenta los efectos de los componentes armónicos de la señal, ya que sus niveles de potencia de señal no se tendrían en cuenta. Un filtro pasabajas es aquel filtro que solo deja pasar las bandas inferiores de frecuencia, particularmente aquella en la que se encuentran distribuidos los componentes de potencia significativos de la señal.

\section{RESULTADOS}

Las señales unipolares sin retorno a cero y con retorno a cero, así como, la señal polar sin retorno a cero, introducen componentes de corriente directa $(\mathrm{dc})$, para $\mathrm{f}=0$, como también, la presencia de los componentes de la función delta para el caso de las señales unipolares, lo que requiere un proceso de filtrado adecuado, para el tratamiento y manejo de dichas señales. Los mayores componentes de magnitud de la densidad espectral de potencia en estas señales se encuentran en dicha frecuencia, por lo que se podría utilizar un filtrado pasabajas para la obtención de tales componentes de frecuencia en los que se concentra la mayor cantidad de potencia de la señal.

En las señales unipolares con retorno a cero y sin retorno a cero, se evidencia la presencia de la componente de corriente directa y además la presencia de los componentes de la función impulso unitario.

En todos los casos, se evidencia la influencia de la señal Senoc (sinc) al cuadrado en las gráficas del espectro de las señales; y particularmente de la función Seno (sin) al cuadrado y la función anterior, en las gráficas del espectro de las señales bipolares con retorno a cero (Bipolar RZ) y las señales Manchester, en la cual se elimina la presencia de la componente de corriente directa.

\section{CONCLUSIONES}

Para el caso 1-A, se encuentran las gráficas del espectro de los códigos de línea de acuerdo con la teoría para el caso normalizado, según lo planteado en los libros de texto que abarcan esta temática. También se verifica que los valores de la frecuencia normalizada se multiplican por el valor de R, en el eje $\mathrm{x}, \mathrm{y}$ los de la densidad espectral de potencia se multiplican por el valor de $\mathrm{Tb}$, en el eje $\mathrm{y}$.

Para el caso 1-B, se siguen manteniendo las características de la función de densidad espectral de potencia, para el caso normalizado, cuando $\mathrm{f}=\mathrm{R}$; lo que se evidencia es el efecto del parámetro Tb en los niveles de excursión de potencia, es decir, cuando la tasa de datos aumenta (R), la frecuencia aumenta (f), pero el tiempo de bit disminuye $(\mathrm{Tb})$, porque es inversamente proporcional a la tasa de datos o frecuencia de datos (fb), por lo cual, los valores de magnitud de la densidad espectral de potencia disminuye, ya que se encuentra en una relación directa con el parámetro tiempo de bit. 
La interfaz de programación y graficación de Python, resulta útil para el análisis de señales, por medio de la interpretación de funciones matemáticas ampliamente utilizadas en los sistemas de comunicación y de codificación de señales.

\section{AGRADECIMIENTOS}

Queremos agradecer a Dios y a la Universidad Distrital Francisco José de Caldas, por habernos acogido y brindarnos la oportunidad de desarrollar nuestra labor docente y poder crecer como personas en la interacción con nuestros estudiantes, compañeros y la comunidad en general. 


\section{REFERENCIAS}

[1] Guerrero, (S). Vanegas, (C), Castang, (G) (2020). Python a su alcance. Bogotá, UD editorial.

[2] Haykin, (S). (2006).Sistemas de Comunicación. Mexico, D.F., MX: Limusa Wiley.

[3] Haykin, (S). Van Veen, (B). (2004). Señales y Sistemas. Mexico, D.F., MX: Limusa Wiley.

[4] Proakis, (J.G). Salehi, (M). (2005). Fundamentals of Communication Systems.New Jersey, USA: Pearson Prentice Hall.

[5] Couch, (L.W). (1998)Sistemas de Comunicaciones Digitales. Mexico, Pearson Prentice Hall. 